\title{
Polonnaruwa Stones Revisited - Evidence for Non-Terrestrial Life
}

\author{
Milton Wainwright ${ }^{2,4}$ and N. Chandra Wickramasinghe ${ }^{1,2,3,4}$ \\ ${ }^{1}$ Buckingham Centre for Astrobiology, University of Buckingham, UK \\ ${ }^{2}$ Centre for Astrobiology, University of Ruhuna, Matara, Sri Lanka \\ ${ }^{3}$ National Institute of Fundamental Studies, Kandy, Sri Lanka \\ ${ }^{4}$ Institute for the Study of Panspermia and Astrobiology, Gifu, Japan
}

\begin{abstract}
Recent discoveries of highly porous low-density carbonaceous asteroids such as 101955 Bennu and Ryugu have motivated a re-examination of the Polonnaruwa stones which fell in central Sri Lanka on 27 December 2012 following a fireball sighting. Previous discoveries of biological entities including fossilized extinct microorganisms (acritarchs) have tended to be discounted as contaminants for the reason that the stones did not fit into a known meteorite category. In view of the new data from space exploration we re-examine samples of the Polonnaruwa stones and confirm earlier evidence for the existence of diatom frustules and other complex biology.
\end{abstract}

Keywords: panspermia, astrobiology, comets, stratospheric microbiota

\section{$1 \quad$ Introduction}

Reports of microfossils in meteorites have a history stretching back over half a century. The earliest claims of microfossils in carbonaceous chondrites by Claus and Nagy (1961) were dismissed as most likely to have arisen from terrestrial contaminants (Anders and Fitch, 1962). Pflug's more careful later studies in the 1980's provided stronger evidence (Pflug, 1984), while Hoover has continued to discover structures in carbonaceous meteorites that have been identified as fossils of cyanobacteria (Hoover et.al., 2011). Despite the growing strength of the evidence, counter claims that such microfossils are probably crystallographic artefacts still dominate the literature. One of the concerns highlighted by critics is that there was no evidence that these meteorites (e.g. Murchison and Orguel) have been in contact with liquid water except perhaps at the time of their formation over 4.5 billion years ago.

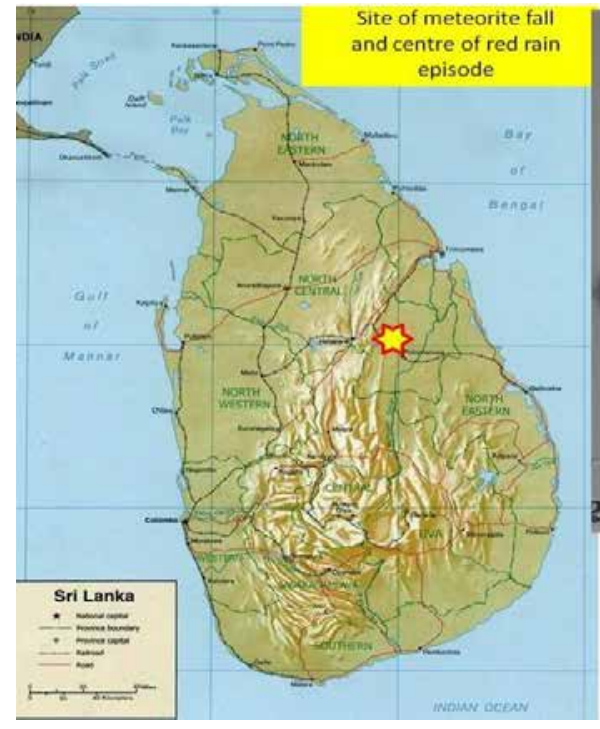

(a)

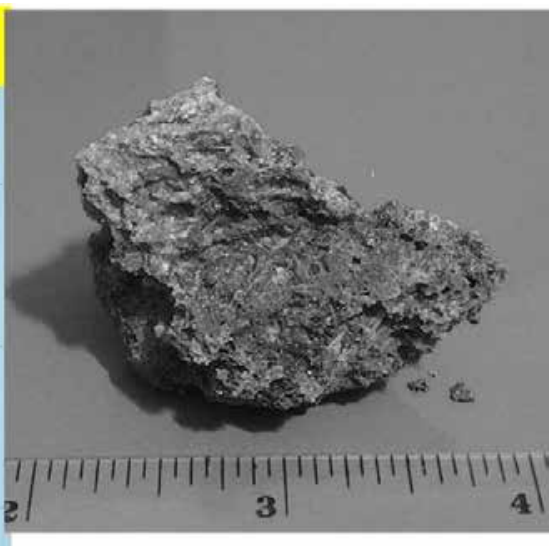

Left Fig 1a Map of Sri Lanka showing location of meteorite

\section{Right Fig 1b Piece of meteorite}

(b)

Figure 1. Polonnaruwa meteorite: a) location of the fall; b) shows a small piece of the meteorite 
Against this backdrop an event that happened in Sri Lanka in 2012 has led to further controversy. On 29 December, 2012 a large meteor appeared in north-central Sri Lanka and was seen to fragment in the sky into many pieces and a spray of meteoroids fell on a rice paddy field located a few miles away from the historic ancient city of Polonnaruwa. The account of this event has been published elsewhere. Fig 1a shows the location of the fall and Fig 1 b shows a small piece of the meteorite.

The meteorite shown in Fig $1 b$ exhibited many characteristics of a carbonaceous chondrite but with a remarkably porous structure. This meteorite on account of its timing was provisionally identified as arising from a cometary fragment in the Taurid complex associated with comet Encke. A few percent carbon as revealed by EDX analysis made it consistent with the properties of carbonaceous meteorites but its exceptionally low density $\left(0.6-1.2 \mathrm{~g} / \mathrm{cm}^{3}\right)$ and high porosity made it significantly different from hitherto classified carbonaceous meteorites, and hence its acceptance as a meteorite had come to be challenged. Not only was it asserted that such low density asteroidal/cometary bodies did not exist, but further it was argued that structures of this type could not survive entry through the atmosphere.

Over the past few years these reservations have been shown to be unjustified and the meteoritic/cometary origin of the Polonnaruwa stones consequently appears to be vindicated (Turner et al, 2020). Analysis of short-lived uranium isotopes in carbonaceous chondrites has yielded excesses of 234uranium over 238-uranium, and 238-uranium over 230-thorium from which it could be concluded that a fluid condition must have persisted in the parent bodies of these meteorites as recently as during the past few 100,000 years. These new results give strong support to the idea that viable microbes including diatoms, and other complex biology (see below) could be carried within fragments of carbonaceous comets.

\section{$2 \quad$ Sampling of Asteroids}

Direct sampling of some asteroids has also unravelled a similar picture. The existence of water and organic molecules has been confirmed on the S-type asteroid 25143 Itokawa (Chan et al., 2020) and on the highly porous C-type carbonaceous asteroid 101955 Bennu (Simon et al., 2020, Nuth et al., 2020). The Hyabusa 2 spacecraft launched by JAXA has further carried out in-situ infrared observations of the dark C-type asteroid 162173 Ryugu which clearly displays characteristics indistinguishable from CI carbonaceous meteorites. However, 162173 Ryugu turned out to be drastically different in its properties from all known meteorites. Most remarkably it possesses an exceptionally low density of $0.8-1.29 \mathrm{~g} \mathrm{~cm}^{-3}$ and a high porosity of $41-55 \%$.

Because of the extremely low tensile strength $(200-280 \mathrm{kPa})$ that has been calculated by Grott et al. (2019) for Ryugu it has been claimed (in our view incorrectly) that any meteoroid with these characteristics would be destroyed on entry through the Earth's atmosphere. We challenge this assertion on the basis of one example that we discuss in this paper - the Polonnaruwa stones of exceptionally low-density $\left(0.6-1.2 \mathrm{~g} / \mathrm{cm}^{3}\right)$ that unquestionably fell from the skies in North Central Sri Lanka on December 29, 2012 following a well attested fireball sighting. Our initial studies of this meteorite showed clearly the presence of embedded microbial structures, and analysis of oxygen isotope studies established it as of non-terrestrial origin (Hoover et al.,2020, Wickramasinghe et al., 2013, Wallis et al. 2013). Theoretical calculations have shown that fragile vesicular meteoroids of the density of the Polonnaruwa/ Aralaganwila stones, as well as of the Ryugu boulder could indeed survive transit through Earth's atmosphere. In this paper we present further evidence of fossil diatom structures integral to the stones, and provide further arguments for their parent body being a repository of microbial life.

Large bodies of liquid water within which lifeforms could develop have been considered to be confined to large planetary bodies such as the Earth with the energy for liquefaction being supplied either from stellar sources (e.g. the sun) or from long-lived radioactive heat sources. Thus, in the solar system the inner planets and some of the Jovian moons present themselves as prime candidates. The argument against the identification of fossils of microorganisms in carbonaceous meteorites has been largely based on the premise that these meteorites were not derived from planetary bodies that had liquid water domains persisting over the billions of years. Moreover, it has been argued that there was little or no evidence for meteorites such as the Murchison meteorite being in contact with liquid water, except perhaps at the time of their formation over 4.5 billion years ago. It is on this basis that the identification of mi- 
crobial fossils in carbonaceous meteorites have been discounted over several decades (Pflug, 1984, Hoover, 2005, 2011)

\section{Re-Examination of Polonnaruwa Stones}

On arrival, the presumptive Polonnaruwa meteorite sample was immediately sterilized with bleach $(10 \% \mathrm{w} / \mathrm{v})$, washed with sterile distilled water and then dried (370C). A portion was then sectioned and examined under the scanning electron microscope, with EDAX attached. Using a hotplate, the sample was first fixed to a polymer stub (using wax) which was then staged in the wire saw setup (Well 3241 Wire Saw). The wire used was approximately $0.17 \mathrm{~mm}$ thick and coated with very fine diamond particles of mode size, circa 30 microns. After the sample was cut, one half was placed in a staging chamber with the face to be analysed flush to the base. Konductomet phenolic mounting compound (20-3375-016) was used to stage the sample. Surface grinding and then polishing of the sample surface is usually undertaken at this point however, in this case only an instantaneous grinding process was conducted. This was done to remove any build up that might be present on top of the surface to be studied and to ensure that only fresh sample material was exposed. The coarseness of pile used was 120 microns using a Bueler Automet 250 for 5 seconds with a touch force of 20N, a head speed of 50 RPM and a Platen speen of 140 RPM. Due to the relatively low conductive nature of the samples and in order to minimise charging effects and optimise image acquisition the samples was coated in gold using a gold sputter coater. The sample was coated for a deposition duration of 1 minute at 15 milliamps. Before being introduced into the SEM (JEOL 6500F) the sample was placed in a vacuum chamber overnight to remove any remaining moisture. Finally, where appropriate, the samples were analysed using EDAX.

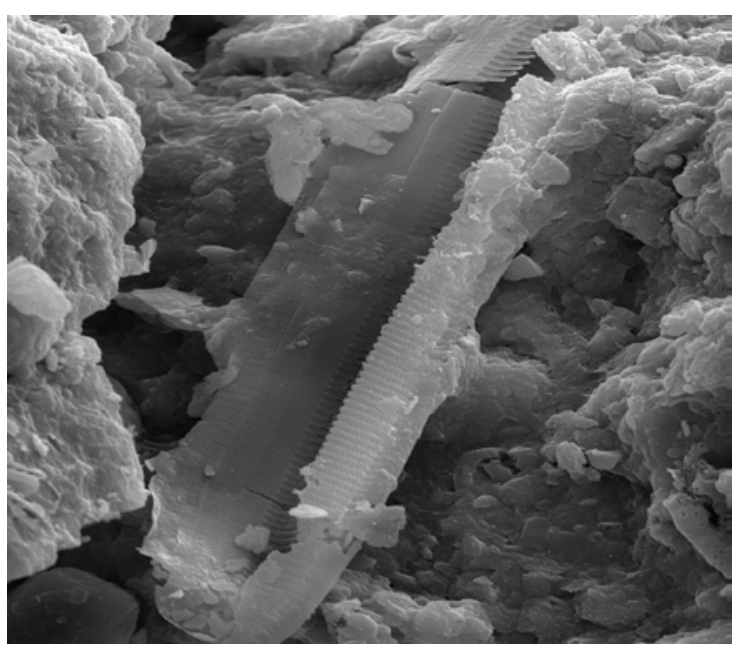

(a)

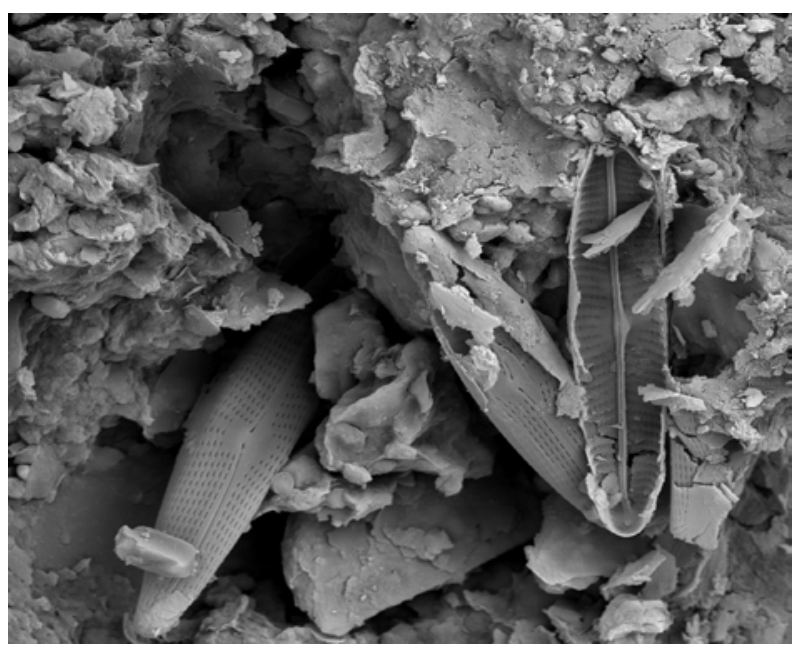

(b)

Figure 2. Diatoms embedded in the surface of a cut section of the Polonnaruwa meteorite (the vertical pennate diatom shown in (a) is circa 25 microns in length). Note that these images are taken from a cut section.

Figure 2 shows clear images of diatom frustules located within the Polonnaruwa meteorite. These are partially integrated within the matrix material and are therefore fossilised and do not result from ingress of modern frustules; even clearer evidence of such integration can be seen by referring to Wickramasinghe et al. (2013). The frustules are identical to modern terrestrial diatoms. Critics would doubtless maintain that diatoms originating from space must be radically different from those found on Earth a point which is further discussed below. The obvious response to these images is that they are modern frustules which have somehow fallen on the cut surface of the meteorite or have gained ingress when the meteorite was possibly submerged in water. The likelihood of the former is negligible and is dismissed by the presence of integration, while we conclude that it is impossible for them to have passed though the small pores to reach the inside of the meteorite; the large erect frustule is 25 microns in length while the background pores are far smaller. An internet critic claimed that the frustules shown in the original re- 
port of the presence of diatoms in the Polonnaruwa meteorite are too pristine to have made an interstellar journey. It is important to note that diatom frustules are extremely hardy. They have for example, been found in 40-50-million-year-old marine Eocene deposits. Frustules also survive intact during the production of filter material, such as Chromosorb which is diatomite crushed, blended, pressed into brick and fired at $900^{\circ} \mathrm{C}$ and then ground (Bens and Drew, 1967). Diatom frustules have also been shown to partially survive heating to $1100^{\circ} \mathrm{C}$ (Zen and Guden, 2017). It would seem likely therefore that diatom frustules would readily survive the rigours of a cosmic journey when embedded within a meteorite. A major objection to a space origin for these frustules is that they are identical to modern diatoms. Surely, critics argue, they would be different from modern terrestrial specimens, unless, in the unlikely event, they had somehow co-evolved in space. This objection can be answered by again pointing out that modern diatoms are identical to those isolated from Eocene sediments and appear not to have dramatically changed as they evolved during the interim vast time period. It is possible therefore that diatoms have not evolved, but were delivered to Earth aeons ago and have not fundamentally changed since.

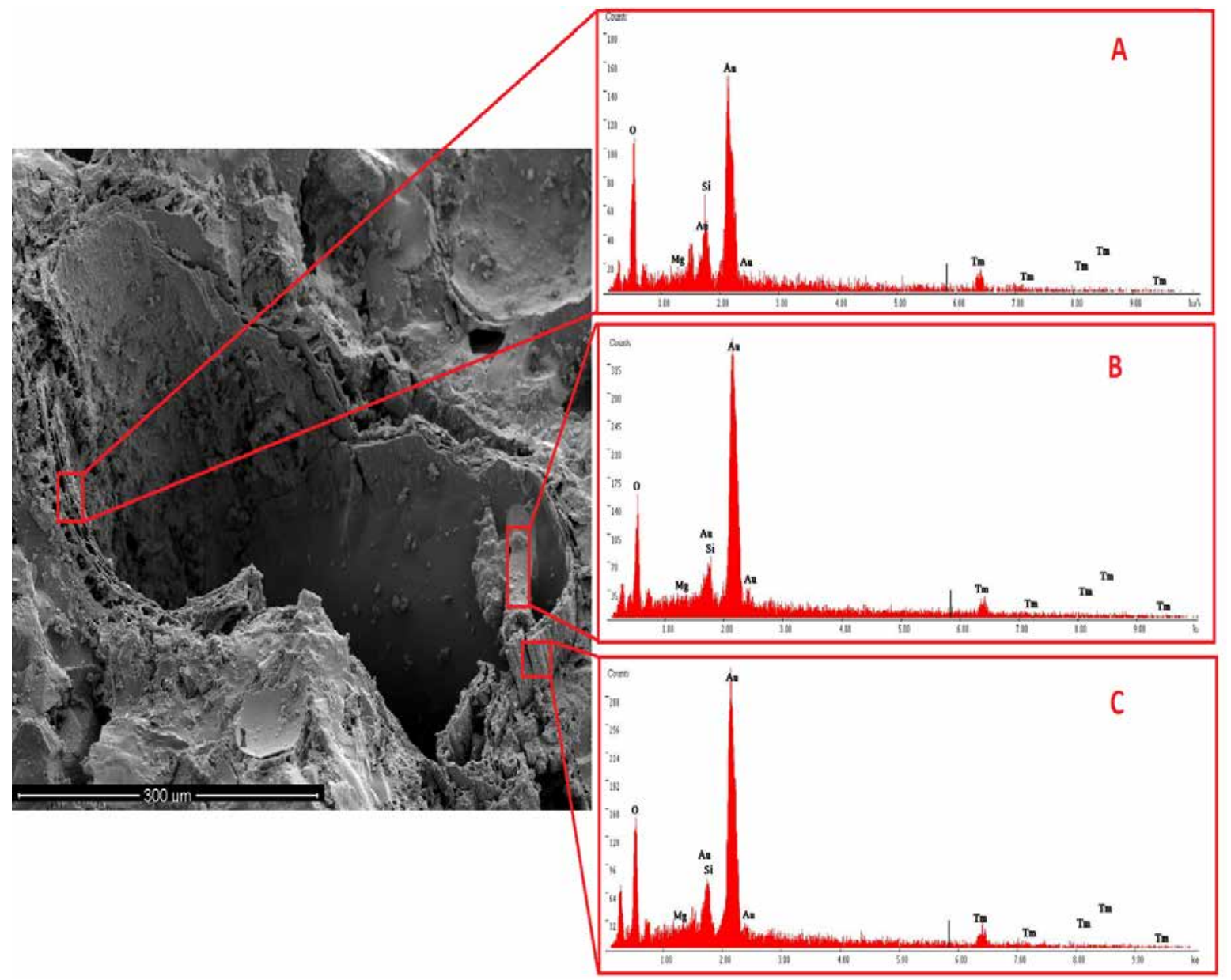

Figure 3. An oval-shaped fossilised biological inclusion and EDAX graph for a complex structure found in the $\mathrm{P}$ meteorite. Note that this image is taken from a cut section.

Figure 3 shows an oval shaped, fossil within the Polonnaruwa meteorite. The oval shaped void contained within the fossil is surrounded by a double-layered structure with cross linkages, suggestive of a plant cell wall (a), (b) parent material and (c) a plant-like palisade layer. EDAX analysis shows that all of the indicated regions have essentially identical composition and are well- integrated in the body of the meteorite. Close-ups of the wall-like structure and palisade layer are shown in Figure 4. 


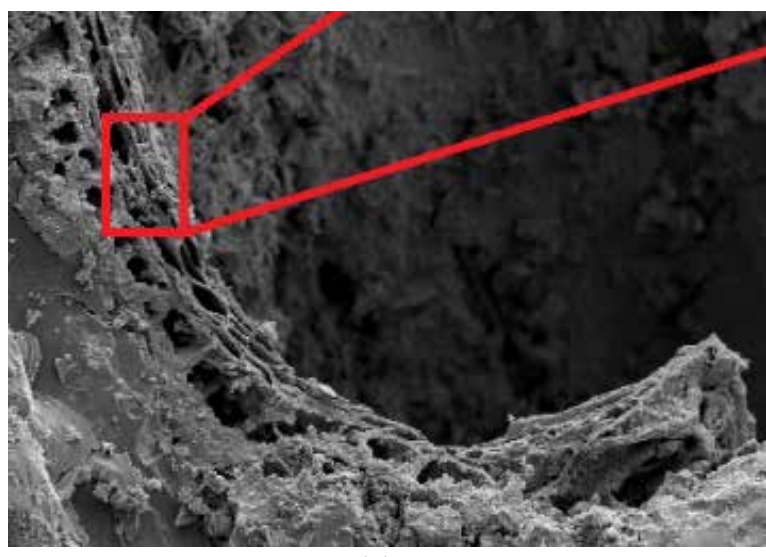

(a)

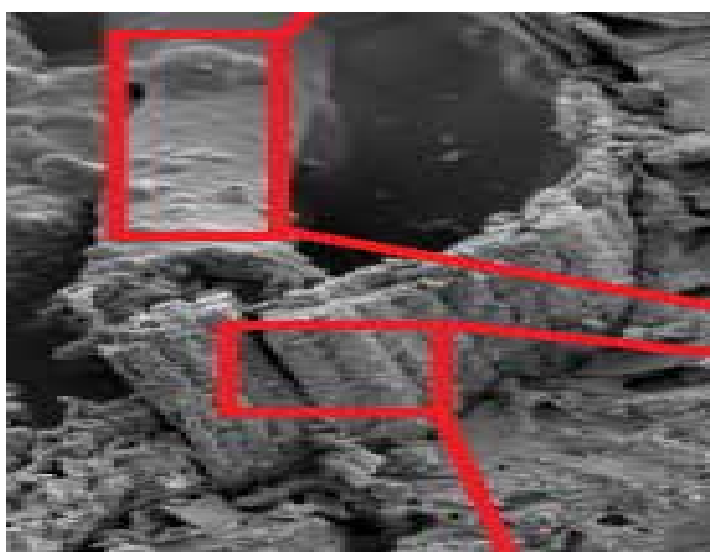

(b)

Figure 4. Detailed images of the fossil wall-like structure (a), and the double plant-like palisade layer (b).

Figure 5 shows a detail of the fossil wall (a) and an image of a present-day grass-shard (b); the similarities are obvious. Although we do not necessarily claim that the meteorite-fossil is a grass shard (its oval structure could, for example, suggest a seed), it is certainly plant-like; it could equally be an unknown biological entity.

The results show that fossils of complex life, including complex eukaryotes exist in the cosmos and that their fossils are delivered to Earth from space; the obvious corollary being that life is, or was, at some time present elsewhere in the Universe. We have also published evidence showing that biological entities are delivered to Earth from space on a continuous basis (Wainwright, 2015).

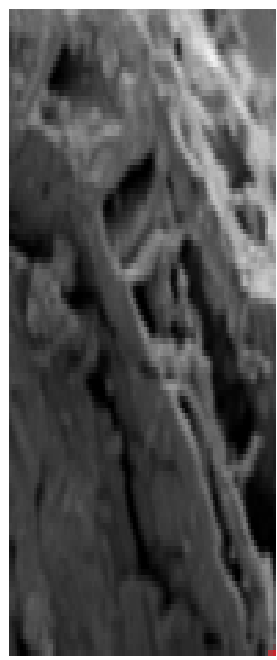

(a)

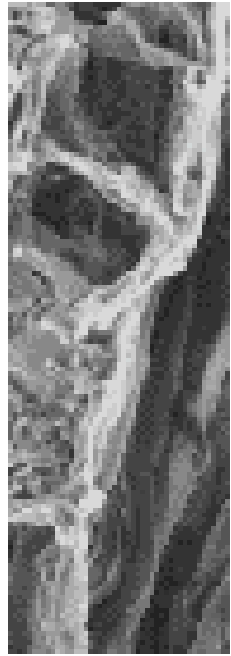

(b)

Figure 5. (a) E/M detail of the compartmentalised wall of the fossilised biological inclusion and (b) E/M detail of a part of the wall of a present-day terrestrial grass shard.

\section{Conclusions}

Our conclusions, which are based on experimental evidence are likely to prove difficult to accept. Some might assert that we are suffering from pareidolia when interpreting plant-like fossil images, a criticism which we have no additional analytical evidence to refute. This argument is not however, tenable in relation to the observed frustules, which are irrefutably diatoms. While it could be suggested that the diatom frustules are terrestrial and washed into the centre of the stone (despite their large size relative to the pore sizes in the bolide) the same argument cannot be used in relation to the plant-like fossil which is clearly integrated into the body of the rock. Nor obviously could this region have fossilised be- 
tween the bolide being observed and it being collected and studied. A terrestrial origin for the fossilized biology described here could possibly be explained by the suggestion made by Urey (1962), who concluded that the most probable origin of supposed biology in the Orgueil meteorite is that a nearby planetary body, at some point, became temporarily contaminated with water and life forms from Earth these having being preserved and are now returning. Whether the introduction of such a currently untestable proposition helps in the matter is, however, debatable. Most scientists will, despite the evidence presented here, likely fall back on parsimony and assert that the Polonnaruwa bolide is not a meteorite and that our findings relate to terrestrial fossils present in a tektite, or other geological sample (as yet, however, we have failed to find any evidence suggesting the presence of biology in tektites) (Chandrajith et al., 2004). Most biologists, like the first named author, who are immersed in an Earth-centred evolution-theory paradigm will find it hard to accept the conclusions we have reached, particularly in regard to our claims that a meteorite contains a plant-fossil, a finding which we claim demonstrates the existence of non-terrestrial life. However, as the fictional detective Sherlock Holmes famously pointed out: "When you have eliminated all which is impossible, then whatever remains, however improbable, must be the truth".

\section{References}

1. Anders, E. and Fitch, F., (1962). Search for organized elements in carbonaceous chondrites. Science 138, 1392

2. Bens, E.M. and Drew, C.H. (1967). Diatomaceous Earth: scanning electron microscope of Chromosorb. Nature 216, 1046-1048.

3. Chan, Q.H.S., Stephant, A., Franchi, I.A. et al (2020). Organic matter and water from the asteroid Itokawa Geochimica et Cosmochimica Acta 71, 4380-4403 (DOI: 10.21203/rs.3.rs-109379/v1)

4. Chandrajith, R. Senaratne,A. and Guntilake,P.G.L. (2004). Aralaganwila stony material: fused soil or extraterrestrial? Proceedings of the Peradenyia University International Research Session, Sri Lanka. 18, 1537.

5. Claus, G. and Nagy, B., (1961). A microbiological examination of some carbonaceous chondrites. Nature 192, 594

6. Fitch, F.W., Schwarz, H.P. and Anders, E., (1962). Organized elements in carbonaceous chondrites. Nature, 193, 1123

7. Grott, M., Knollenberg, J., Hamm, M. et al (2019). Low thermal conductivity boulder with high porosity identified on C-type asteroid (162173) Ryugu. Nature Astronomy, 3(11):1-6. DOI: 10.1038/s41550-019-0832-x

8. Hoover, R.B., (2005). In R.B. Hoover, A.Y. Rozanov and R.R. Paepe (eds.) Perspectives in Astrobiology, (IOS Press Amsterdam)

9. Hoover, R.B., (2011). Fossils of Cyanobacteria in C11 carbonaceous meteorites: implications to life on comets, Europa and Enceladus. Journal of Cosmology 13: (http://www.panspermia.org/hoovermeteorites.pdf)

10. Hoover R.B., Hoover M.J., Hoyle F., Wickramasinghe N.C. and Al-Mufti S. (1986) Diatoms on Earth, Comets, Europa and in interstellar space. Earth, Moon, and Planets 35, 19-45.

11. Hoover, R. B., Frontasyeva, M. and Pavlov, S. (2020). Epithermal neutron analysis of carbonaceous chondrites and the Polonnaruwa/Araganwila stones. Aspects in Mining and Mineral Science 6, 669-679. doi: 10.31031/AMMS.2020.06.000626

12. Hoover, R. B., Wallis, J.,Wickramarathne, K., Samaranayake, A., Williams, G., Jerman, G., Wallis, D. H., and Wickramasinghe, N. C. (2013) Fossilized diatoms in meteorites from recent falls in Sri Lanka. Proc. SPIE 8965: 8865_06: 1-14. doi:10.1117/12.2028605.

13. Joseph A. Nuth, N.A., Abreu, N., Ferguson, F.T., et al (2020). Volatile-rich Asteroids in the Inner Solar System

14. The Planetary Science Journal, 1:82

15. Pflug, H.D., (1984). Ultrafine structure of organic matter in meteorites", in Fundamental Studies and the Future of Science, C. Wickramasinghe (ed.), Cardiff, University College Cardiff Press.

16. Simon, A.A., Kaplan, H.H., Hamilton, V.E. et al. (2020). Widespread carbon-bearing materials on near-Earth asteroid (101955) Bennu. Science 06 Nov 2020, Vol. 370, Issue 6517, eabc3522

17. Turner, S., McGee, L., Humayun, M. et al. (2021). Carbonaceous chondrite meteorites experienced fluid flow within the past million years. Science $371,164-167$

18. Urey, H.C. (1962). Origin of life-like forms in carbonaceous chondrites. Nature 1931962 1119-23.

19. Wainwright, M. (2015). Biological entities and DNA containing masses isolated from the stratosphere-evidence for a non-terrestrial origin. Astronomical Review 11, 25-50. 
20. Wallis, J., Wickramasinghe, N.C., Wallis, D.H. et al., (2013). Physical, chemical and mineral properties of the Polonnaruwa stones, in Proceedings of SPIE 8865, 886508-1

21. Wickramasinghe, J.T., Wickramasinghe, N.C. and Napier, W.M. (2010). Comets and the Origin of Life, World Scientific Press, Singapore.

22. Wickramasinghe, N. C., Wallis, J.,Wallis, D.H. and Samaranyake, A. (2013). Fossil diatoms in a new carbonaceous meteorite Journal of Cosmology, 21, arxiv1302.2398.

23. Zeren, D. and Guden, M. (2017). Increased compression strength of epoxy resin with the addition of heattreated natural nano-structured diatom frustules. Journal of Composite Materials 51, 1681-1689. 